March 2018

\title{
Turmeric for prevention of dementia: food for thought
}

\author{
Mustafa M. Husain \\ University of Texas Southwestern Medical Center \\ Yamna Channa \\ Aga Khan University \\ Mohan Chilukuri \\ University of Texas Southwestern Medical Center
}

Follow this and additional works at: https://ecommons.aku.edu/pakistan_fhs_mc_mc

Part of the Diseases Commons, and the Food Science Commons

\section{Recommended Citation}

Husain, M. M., Channa, Y., Chilukuri, M. (2018). Turmeric for prevention of dementia: food for thought. The American journal of geriatric psychiatry, 26(3), 278-279.

Available at: https://ecommons.aku.edu/pakistan_fhs_mc_mc/33 


\title{
Turmeric for Prevention of Dementia: Food for Thought
}

\author{
Mustafa M. Husain, M.D., Yamna Channa, M.B.B.S., Moban Chilukuri, M.D.
}

Let thy food be thy medicine, and let thy medicine be thy food.

-Hippocrates.

$\mathrm{T}$ he understanding of neurodegenerative disorders has increased a great deal in the last few decades. Our understanding of the causes, types, and pathophysiology of dementia has increased immensely - but we are still a long way off from preventing or finding treatments for these disorders.

The anti-inflammatory effects of the active compound in the bright yellow South Asian spice turmeric have been documented as early as $1973 .{ }^{1}$ Turmeric has been considered an effective herbal remedy for many health problems, including mental health problems, and is firmly entrenched among the traditional herbal cures in the Indo-Pak subcontinent. It is a component of widely used herbal treatment for cold, catarrh, and cough. ${ }^{2}$ Recent research indicates that the active compound of turmeric, curcumin, may have uses that extend well beyond that of a spice used to flavor food. ${ }^{3}$

Previous research into the effect of turmeric on Alzheimer disease (AD) indicates that it has antioxidant activity and that it inhibits amyloid beta aggregation. These studies recommend further research into the impact of curcumin on dementia. ${ }^{4}$ Earlier research has shown that patients develop predictable changes in brain pathology before symptoms of AD develop. It is also known that tau pathology leads to cognitive deficits in Alzheimer dementia. ${ }^{5}$ Previous research recommended that any future research into the role of curcumin on cognition include measures of cognitive outcome in conjunction with biological markers of neurodegeneration. ${ }^{6}$ Small et al. ${ }^{7}$ fulfill that recommendation.

In this issue of The American Journal of Geriatric Psychiatry, Small et al. ${ }^{7}$ examine the relationship between the use of a highly bioavailable form of curcumin (Theracurmin) and its effects on memory and neuropathological markers. Forty subjects were recruited for this trial; 21 of these received Theracurmin and the remaining subjects received a placebo. The study subjects were screened at baseline for any comorbidity that could interfere with study results. For example, all subjects were screened for major depressive disorder, which can cause pseudo-dementia and thus decrease cognition.

Small et al. recognized the need for a study that would monitor the effect of curcumin on long-term memory and cognition. The period of 18 months was chosen based on the results of previous trials suggesting that this is the optimum time period to monitor long-term effects of curcumin. This placebo-controlled trial recruited individuals without dementia and those with mild cognitive impairment. People with dementia were excluded from the study as the aim was to study the role of curcumin as a prophylactic agent rather than as a treatment or cure for dementia.

Change in memory of study subjects was measured using the Buschke SRT and the Consistent Long Term Retrieval Scores. Changes in biomarkers (i.e., accumulation of amyloid and tau plaques) were monitored via 2-(1-\{6-[(2-[F-18] fluoroethyl) (methyl) amino]-2-naphthyl\} ethylidene) malononitrile positron emission tomography (FDDNP-PET). Buschke SRT is a sensitive test that can predict future cognitive

Received November 15, 2017; accepted November 15, 2017. From the Department of Psychiatry and Behavioral Sciences (MMH), Duke University School of Medicine, Durham, NC; Department of Psychiatry (MMH, MC), University of Texas Southwestern Medical Center, Dallas, TX; and the The Aga Khan University (YC), Karachi, Pakistan. Correspondence and reprint requests to Mustafa M. Husain, M.D., Department of Psychiatry, University of Texas Southwestern Medical Center, 5323 Harry Hines Blvd., Dallas, TX 75390. e-mail: mustafa.husain@ utsouthwestern.edu

(C) 2018 Published by Elsevier Inc. on behalf of American Association for Geriatric Psychiatry.

https:/ / doi.org/10.1016/j.jagp.2017.11.010 
decline. As well as following study subjects for 18 months, Small et al. also used tools that predict future cognitive decline. This allows for accurate interpretation of the long term effects of Theracurmin.

The group using Theracurmin showed improved memory as measured by the Buscke SRT and the Consistent Long Term Retrieval Scores. This group was also found to have decreased FDDNP binding in the amygdala and hippocampus. Thus, the results indicate that Theracurmin improves memory and cognition. The results of this trial by Small et al. also indicate that curcumin decreases the neuropathological accumulation of tau and amyloid proteins in the amygdala and hypothalamus-a finding that is correlated with dementia.

What is the impact of this study on the current treatment, management, and prevention of dementia?

Dementia is a poorly understood disease and there is little evidence supporting definitive cure, treatment, or prevention. This study is based on previously conducted experiments that provide indication for the use of curcumin in disintegrating tau filaments and amyloid plaques. $^{8}$

Small et al.'s investigation is an important and novel study that opens doors for future targeted intervention in the field of dementia treatment and prevention research. Theracurmin is a natural herbal compound and is considered safe compared to other pharmacological agents that are standard of care for dementia currently. The side effects reported in this trial by Small et al. involve mild gastrointestinal side effects. No subjects reported side effects severe enough to require discontinuation of Theracurmin. For any patient population this is an important aspect to consider, but more so in patients suffering from dementia. These patients are older and are often victims of the negative effects of polypharmacy. Therefore the fact that Theracurmin has few side effects is an important advantage. It would also be relevant for future research to monitor possible drug interactions of Theracurmin with other medications that are commonly used in the elderly patient population.

Future studies may also clarify if the effect of Theracurmin to delay the onset of dementia or to prevent it. This is an important consideration, as Theracurmin may have the potential to significantly decrease the incidence and prevalence of this debilitating illness.

Small et al.'s findings also bolster the results of previous research that indicates an improvement in mood and anxiety symptoms with the use of curcumin. It would be an interesting area for future research to develop a trial focused on illuminating further this aspect of the effects of curcumin.

\section{References}

1. Srimal RC, Dhawan BN: Pharmacology of diferuloyl methane (curcumin), a non-steroidal anti-inflammatory agent. J Pharm Pharmacol 1973; 25:447-452

2. Vohara SB: Unani joshanda drugs for common cold, catarrh, cough and associated fevers. J Ethnopharmacol 1986; 16:201-211

3. Zdrojewicz Z, Szyca M, Popowicz E, et al: [Turmeric-not only spice]. Pol Merkur Lekarski 2017; 42:227-230

4. Mirmosayyeb O, Tanhaei A, Sohrabi HR, et al: Possible role of common spices as a preventive and therapeutic agent for Alzheimer's disease. Int J Prev Med 2017; 8:5

5. Bejanin A, Schonhaut DR, La Joie R, et al: Tau pathology and neurodegeneration contribute to cognitive impairment in Alzheimer's disease. Brain 2017; 40:3286-3300
6. Rainey-Smith SR, Brown BM, Sohrabi HR, et al: Curcumin and cognition: a randomised, placebo-controlled, double-blind study of community-dwelling older adults. Br J Nutr 2016; 115:21062113

7. Small G, Siddarth P, Li Z, et al: Memory and brain amyloid and tau effects of a bioavailable form of curcumin in nondemented adults: a double-blind, placebocontrolled 18-month trial. Am J Geriatr Psychiatry 2018; 26:266-277

8. Rane JS, Bhaumik P, Panda D: Curcumin inhibits tau aggregation and disintegrates preformed tau filaments in vitro.J Alzheimers Dis 2017; 60:999-1014 